in hIL4-10FP group there was no enhanced cartilage degeneration detected compared to the PBS group (fig 3 ).

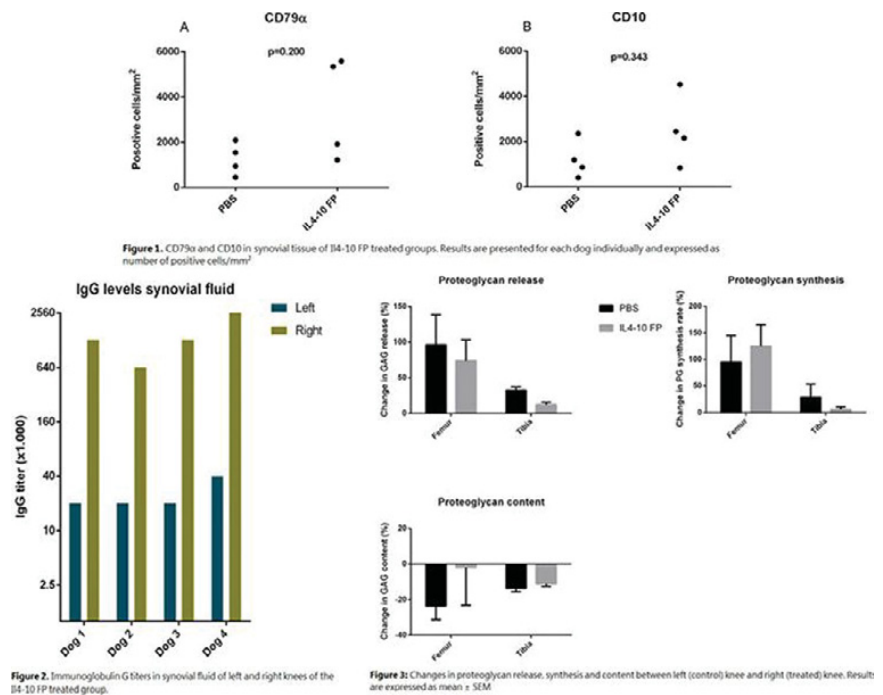

Conclusions: Repetitive intra-articular injection of human IL4-10FP led to antibody formation in a non-inflammatory canine model of OA. Despite the immune response, proteoglycan turnover parameters were comparable between the two treatment groups, suggesting a beneficial effect of hIL4-10FP. This study also shows that it is not evident to use a human protein in a (canine) animal model, although this is often done. Instead, a species specific protein is warranted. Therefore a canine version of IL4-10FP will be developed to study its DMOAD activity in this model.

References:

[1] van Roon JA, Lafeber FP, Bijlsma JW. Synergistic activity of interleukin-4 and interleukin-10 in suppression of inflammation and joint destruction in rheumatoid arthritis. Arthritis and rheumatism. 2001;44(1):3-12.

Disclosure of Interest: None declared

DOI: 10.1136/annrheumdis-2017-eular.1498

\section{FRI0009 ACCELERATED DEVELOPMENT OF AGING-ASSOCIATED AND INSTABILITY-INDUCED OSTEOARTHRITIS IN 12/15-LIPOXYGENASE DEFICIENT MICE}

L. Habouri, F. El Mansouri, Y. Ouhaddi, J.-P. Pelletier, J. Martel-Pelletier, M. Benderdour, H. Fahmi. Medicine, CRCHUM, Montreal, Canada

Background: 12/15-Lipoxygenase (12/15-LOX) catalyzes the generation of various anti-inflammatory lipid mediators, and has been implicated in several inflammatory and degenerative diseases. However, there is currently no evidence that 12/15-LOX has a role in osteoarthritis (OA)

Objectives: The aim of this study was to investigate the role of 12/15-LOX in the pathogenesis of $O A$

Methods: The development of aging-associated and destabilization of the medial meniscus (DMM)-induced OA were compared in 12/15-LOX-deficient (12/15-LOX/-) and wild-type (WT) mice. The extent of cartilage damage was evaluated by histology. The expression of OA markers was evaluated by immunohistochemistry and RT-PCR. Cartilage explants were stimulated with IL-1 $\alpha$ in the absence or presence of the 12/15-LOX metabolites, 15-HETE, 13-HODE or LXA4, and the levels of MMP-13, NO and PGE 2 were determined. The effect of LXA4 on the progression of OA was evaluated in WT mice.

Results: The expression of 12/15-LOX in cartilage increased during the progression of DMM-induced OA and with aging in WT mice. Cartilage degeneration was more severe in 12/15-LOX-/- mice compared to WT mice in both models of OA, and this was associated with increased expression of MMP-13, ADAMTS5, iNOS, and mPGES-1. Treatment of cartilage explants with 12/15-LOX metabolites, suppressed IL-1 $\alpha$-induced production of MMP-13, NO and $\mathrm{PGE}_{2}$, with LXA4 being the most potent. Intra-peritoneal injection of LXA4 reduced the severity of DMM-induced cartilage degradation.

Conclusions: These data demonstrate an important role of 12/15-LOX in OA and suggest that activation of this pathway may provide a novel strategy for prevention and treatment of $O A$.

Acknowledgements: This work was supported by the Canadian Institutes of Health Research (CIHR) Grant MOP-130293, the Arthritis Society, and the Fonds de la Recherche du Centre de Recherche du Centre Hospitalier de l'Université de Montréal (CRCHUM).

Disclosure of Interest: None declared

DOI: 10.1136/annrheumdis-2017-eular.6184

\section{FRI0010 METABOLIC DYSREGULATION ACCELERATES JOINT DEGENERATION UPON MECHANICALLY INDUCED CARTILAGE DAMAGE, DRIVEN BY LOCAL INFLAMMATION; AN IN VIVO RAT STUDY}

H.D. Visser ${ }^{1,2}$, S. Mastbergen ${ }^{2}$, A. Kozijn ${ }^{2}$, K. Coeleveld ${ }^{2}$, M.V. Rijen ${ }^{1}$, H. Weinans ${ }^{1,2,3}$, F. Lafeber ${ }^{2}$. ${ }^{1}$ Orthopaedics; ${ }^{2}$ Reumatology \& Clinical Immunology, UMC Utrecht, Utrecht; ${ }^{3}$ Biomechanical Engineering, Delft University of Technology, Delft, Netherlands

Background: Obesity is a well-known and important risk factor for osteoarthritis (OA). Moreover, obesity is highly associated with the metabolic syndrome (MetS) ${ }^{1}$. Growing evidence indicates that both $\mathrm{OA}$ and MetS are low-grade inflammatory conditions with elevation in systemic inflammatory markers. Nonetheless, it is unclear whether MetS low-grade inflammation induces OA, or contributes to the disease.

Objectives: To determine the contribution of metabolic alterations, induced by a High-Fat Diet (HFD), on the onset or progression of OA in a rat model of local cartilage damage.

Methods: Forty Wistar rats (12 weeks old, male), were randomly divided over two groups: twenty rats were fed a HFD $(60 \%$ of the kcal contained fat:D12492i, Research Diets Inc.) while the other animals received a standard diet. After 12 weeks, local articular cartilage damage was induced on the femoral condyles, in one knee joint according to the groove model in 14 rats of each diet group. Remaining animals served as a control group in each arm. At week 24, serum was collected, subchondral bone was assessed by $\mu$ CT scan (Quantum FX, PerkinElmar,USA), OA severity was evaluated by rat OARSI histopathology score and macrophage presence with CD68 immunostaining from histological sections was assessed. Results: HFD feeding resulted in metabolic dysregulation as indicated by significantly increased metabolic parameters (weight, fasting insulin and total cholesterol) compared to the standard fed rats. HFD feeding alone resulted in mild cartilage degeneration ( $2 \pm 1.1$ vs $0.58 \pm 0.7 ; p=0.06)$ and synovial membrane inflammation $(1.0 \pm 0.6$ vs $0.3 \pm 0.5 ; p=0.075)$ both subscores of the rat OARSI histopathology score. However, when HFD feeding is combined with the surgical model of applied local cartilage damage, OA severity is statistically significant increased compared to the local cartilage damage group on a standard diet $(6.2 \pm 2.1$ vs $3.4 \pm 1.4 ; p=0.001)$. Synovial membrane inflammation $(1.3 \pm 0.9 \mathrm{vs}$ $0.5 \pm 0.5 ; p=0.011$ ) and multiple large osteophyte formation, demonstrated by histology $(0.9 \pm 1$ vs $0.2 \pm 0.4 ; \mathrm{p}=0.04)$ and quantified on $\mu \mathrm{CT}\left(328 \pm 349 \mu \mathrm{m}^{3}\right.$ vs $\left.7 \pm 14 \mu \mathrm{m}^{3} ; \mathrm{p}=0.0001\right)$, contributes most to this increased $\mathrm{OA}$ severity. Immunohistochemical CD68 expression as observed on both the synovial membrane as well as in the subchondral bone and around the formed osteophytes can explain the increase in selected inflammatory parameters when groove surgery is combined with a HFD (Figure 1).
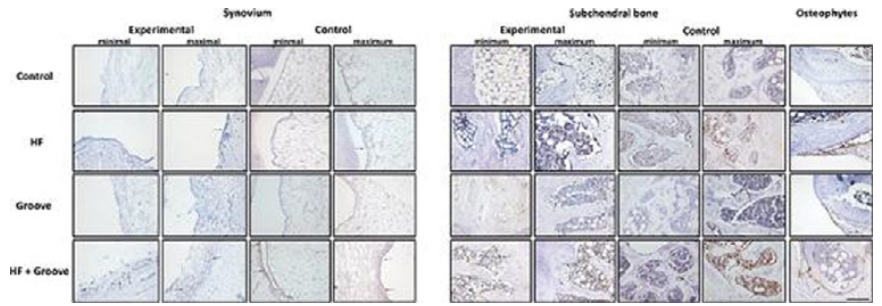

Conclusions: This study shows that a HFD induces metabolic alterations and increases the inflammatory state of the joint. This by itself does not result in severe OA. However, when adding a HFD to a mild cartilage damage model of OA, joint degeneration is significantly increased. This progression of joint degeneration appears to be driven mainly by inflammatory responses as demonstrated by an increased CD68 expression in both the subchondral bone and synovium membrane with increased osteophytosis. Hence, our findings indicate that systemic metabolic and subsequent inflammatory factors need an additional trigger to contribute to the progression of the OA.

References:

[1] Zhuo Q, Yang W, Chen J and Wang Y, Metabolic syndrome meets osteoarthritis. Nat Rev Rheumatol, 2012. 8(12): p. 729-37.

Disclosure of Interest: None declared

DOI: 10.1136/annrheumdis-2017-eular.1639

\section{FRI0011 TARGETING NEUTROPHIL MICROVESICLES TO DAMAGED CARTILAGE USING ANTIBODIES TO POST TRANSLATIONALLY MODIFIED COLLAGEN II}

L.M. Topping, H. Rhys, L. Norling, A. Nissim. Biochemical Pharmacology, William Harvey Research Institute, Queen Mary University of London, London, United Kingdom

Background: Microvesicles (MV) are double membrane-bound extracellular vesicles released from the plasma membrane of cells. MV derived from polymorphonuclear neutrophils (PMN) promote tissue protection, and have been demonstrated to penetrate cartilage during inflammatory arthritis and provide protection to the tissue ${ }^{1}$. 